\title{
IMRT Optimization with Both Fractionation and Cumulative Constraints*
}

\author{
Delal Dink ${ }^{1}$, Mark Langer ${ }^{2}$, Seza Orcun ${ }^{3}$, Joseph Pekny ${ }^{1}$, Ronald Rardin ${ }^{4}$, \\ Gintaras Reklaitis ${ }^{1}$, Behlul Saka ${ }^{4}$ \\ ${ }^{1}$ School of Chemical Engineering, Purdue University, W. Lafayette, USA \\ ${ }^{2}$ Department of Radiation Oncology, Indiana University, Indianapolis, USA \\ ${ }^{3} e$-Enterprise Center, Discovery Park, Purdue University, W. Lafayette, USA \\ ${ }^{4}$ Department of Industrial Engineering, University of Arkansas, Fayetteville, USA \\ E-mail: bsaka@uark.edu
}

Received June 8, 2011; revised June 30, 2011; accepted July 23, 2011

\begin{abstract}
Radiation therapy plans are optimized as a single treatment plan, but delivered over 30 - 50 treatment sessions (known as fractions). This paper proposes a new mixed-integer linear programming model to simultaneously incorporate fractionation and cumulative constraints in Intensity Modulated Radiation Therapy (IMRT) planning optimization used in cancer treatment. The method is compared against a standard practice of posing only cumulative limits in the optimization. In a prostate case, incorporating both forms of limits into planning converted an undeliverable plan obtained by considering only the cumulative limits into a deliverable one within $3 \%$ of the value obtained by ignoring the fraction size limits. A two-phase boosting strategy is studied as well, where the first phase aims to radiate primary and secondary targets simultaneously, and the second phase aims to escalate the tumor dose. Using of the simultaneous strategy on both phases, the dose difference between the primary and secondary targets was enhanced, with better sparing of the rectum and bladder.
\end{abstract}

Keywords: IMRT, Mixed-Integer Linear Programming, Optimization, Cumulative Dose Constraints, Fractionation, Two-Phase Planning, Uniform Fractionation

\section{Introduction}

The success of radiation treatment rests on satisfying dose limits within critical or healthy structures. These criteria are determined mostly after years of experience and increasingly by formal clinical trials. Strictly, the prescriptions should be stated both in terms of cumulative doses as well as the dose received per treatment session known as fraction [1,2]. Both cumulative dose and per-fraction dose limits appear in the historical reports and clinical trial protocols that form the experience upon which modern treatments are based.

In practice, planning optimization is based on cumulative dose limits alone. Numerous methods have been proposed in the literature to generate radiation therapy

\footnotetext{
*Supported in part by grants from the National Science Foundation (ECS 020145, CMMI 0813896) and from the National Institutes of Health (1R41 CA91688-01) and the Indiana $21^{\text {st }}$ Century Fund grant 83001403
}

plans. Of these methods, optimization models using mathematical programming formulations have been developed to determine the best beamlet intensities [3-11] and the best aperture intensities $[12,13]$. Further methods include randomized approaches, such as simulated annealing [14-17] and genetic algorithms [18-20], and non-linear gradient techniques [21-24].

This paper investigates a conventional approach of optimizing over only cumulative limits as the optimization constraints alone and then dividing the plan into integral number of fractions after the optimization in order to obtain the daily delivery plan. We refer to this approach as Cumulative First Method. Then we present a new Simultaneous Method approach including both cumulative and fraction size limits simultaneously in the optimization which guarantee a plan that can be equally fractionated into a uniform course of the treatment. Both of the approaches try to achieve the uniform fractionation plan, where the same doses are delivered in every fraction. 
The ability of the proposed approach to generate a solution that simultaneously satisfies the cumulative dose constraints and the fraction size constraints is tested via a test case, and the effect of not including the fraction sizes on the obtained objective, the minimum tumor dose and the normal structures is evaluated. By imposing both the cumulative and the fraction size limits into the formulation explicitly, the Simultaneous Method guarantees a solution when any feasible one exists by searching the feasible solution space with the fractionation criteria included.

In addition to the two uniform fractionation methods, we also consider a two-phase approach familiar in conventional practice which uses different uniform fractions in two successive periods of treatment. The primary and secondary targets are covered as uniformly as possible during the first phase of the treatment using the fraction sizes traditionally accepted for disease sterilization. Then, a second phase is added with the main focus to escalate the primary target. Relaxing the secondary target minimum fraction size requirements partially in the second phase of the treatment provides two potential advantages to the two-phase planning approach over the uniform fractionation: escalating the tumor dose or dropping the normal tissue exposure, if not both. Here, these potential advantages will be explored in Intensity Modulated Radiation Therapy (IMRT) planning where highly nonuniform beam patterns are possible by dividing the beam faces into small beamlets or bixels.

There have been studies where a non-IMRT module is accompanied by IMRT in the second phase for tumor boosting [25-27]. Two-phase IMRT planning was also tested where the coverage volume is changed from whole pelvic irradiation to prostate only in the second phase and it was concluded that when the whole pelvic area is simultaneously irradiated with IMRT higher dose to primary target and lower dose to the subclinical disease can be achieved [28]. Simultaneous optimization of the twophases for IMRT was addressed in [29] by using a gradient search algorithm with cumulative dose constraints alone.

Here, we show the advantages of adapting our Simultaneous Method to two-phase planning. A two-phase IMRT plan by irradiating the whole pelvic area is obtained with one-step optimization, where the irradiated volume is kept the same but the dose constraints for the two-phases are different and the dose difference between the primary disease and the secondary target is enhanced. The cumulative minimum dose limits on the secondary targets are satisfied during the first phase of the treatment. The goal of the second phase is solely to boost the tumor dose given the irradiated volume remains as the whole pelvic. In the second phase, it is no longer the interest of the planner to satisfy the minimum fraction size requirements of the secondary target since the potential disease on those sites are already eliminated during the first phase. Of course, the fraction sizes on the organs at risk remain to be a concern in the second phase.

This paper is organized as follows. Section 2 develops all the methods including Cumulative First Method, Simultaneous Method, and Two-Phase Planning with Simultaneous Method used to generate treatment plans in this paper. The results from computed plans are presented in Section 3 on a prostate case. Finally, the conclusions and possible future research directions are given in Section 4.

\section{Methods and Materials}

In the formulations, each structure is identified as a primary target, a secondary target, or an organ at risk. The set of normal tissue indices is denoted by $K$, and the set of indices for the secondary target is denoted by $V$. The set of tissue points drawn from the primary target is denoted by $T$, that drawn from the $k$-th secondary target is denoted as $S_{k}$, and that drawn from the $k$-th organ at risk is denoted as $H_{k}$.

The solution approach in all cases employed a beamlet-based optimization in which dose to any point could be expressed as a linear combination of the individual beamlet intensities. Thus, dose $d_{i}$ is the dose received at tissue point $i$ in the system is defined as

$$
\begin{aligned}
& \sum_{g \in G} \sum_{j \in J^{g}} a_{i j g} x_{j g}=d_{i} \\
& \forall i \in T \bigcup\left\{S_{k}: k \in V\right\} \bigcup\left\{H_{k}: k \in K\right\}
\end{aligned}
$$

where $x_{j g}$ is the value of total intensity assigned to beamlet $j$ of angle $g$, and $a_{i j g}$ are pre-computed dose coefficients at point $i$ from beamlet $j$ of beam $g$.

The chosen objective function in all approaches is to maximize the average tumor dose, a metric which has been found to be a predictor of tumor persistence after radiotherapy. It is represented as in (2) where $|T|$ represents the total number of tumor points.

$$
\text { Maximize } \sum_{i \in T} \frac{d_{i}}{|T|}
$$

In addition, preliminary work showed that the average tumor dose to be a computationally efficient surrogate for the minimum tumor dose in the presence of a strong tumor-dose homogeneity constraint. Both the value of the objective function and the value of the minimum tumor dose are evaluated in the results.

A homogeneity limit, defined as the ratio of the minimum to the maximum dose received, is enforced in all models to sustain a near uniform tumor dose profile. 


$$
d_{\min } \leq d_{i} \leq \frac{d_{\min }}{\alpha} \quad \forall i \in T
$$

Here $d_{\min }$ represents the minimum tumor dose value and parameter $\alpha>0$ is the allowed ratio.

An alternative way of enforcing homogeneity in the tumor instead of specifying a ratio parameter $\alpha$ between the minimum and maximum tumor doses is to impose strict lower and upper bounds $l_{\text {tumor }}$ and $u_{\text {tumor }}$, in the tumor, and replace relations (3) with (4) and (5).

$$
\begin{array}{ll}
d_{i} \geq l_{\text {tumor }} & \forall i \in T \\
d_{i} \leq u_{\text {tumor }} & \forall i \in T
\end{array}
$$

\subsection{Cumulative First Method}

This conventional approach is based on the assumption that, if one can find a good plan to satisfy the cumulative dose limits, an appropriate number of fractions to indirectly satisfy the fraction size limits and to deliver the plan exists. Constraint sets (6) and (7) ensure that the maximum cumulative dose constraints for all healthy tissue points and minimum cumulative dose constraints for all secondary target points are satisfied. The superscript "total" represents the cumulative dose limits in the relations below. The limits $l_{\text {tumor }}$ and $u_{\text {tumor }}$ in the constraint sets (4) and (5) continue to represent the cumulative lower and upper bounds for the tumor.

$$
\begin{gathered}
d_{i} \leq u_{i k}^{\text {total }} \quad \forall i \in H_{k}, k \in K \\
d_{i} \geq l_{k}^{\text {total }} \quad \forall i \in S_{k}, k \in V
\end{gathered}
$$

Sometimes, dose volume limits are specified to limit the fractional portion $p$ of a structure volume that can exceed a threshold dose in a normal tissue. A layering heuristic employed here to incorporate these limitations in the above model is as follows: After ordering the optimization points of the tissues with partial volume limits according to their distance to the tumor margin in $3 \mathrm{D}$, the upper dose limits are assigned to that fraction $p$ of the points that lie closest to the target boundary, and the lower dose limits are assigned to $(1-p)$ fraction of those points farthest from the target boundary [30].

Once a plan is developed based only on cumulative dose limits, it still must be divided into a fixed number of fractions which in today's practice are taken to be uniform. Practical bounds on $N$ can be developed for the maximum and minimum number of allowed uniform fractions. An upper bound $\bar{N}$ comes from the minimum total doses achieved by tumor and secondary targets and dividing these numbers by the minimum allowed daily dose that should be delivered to each. The minimum of these ratios results in the upper bound for number of fractions given below in (8). Similarly, the lowest number $\underline{N}$ of allowed fractions is governed by the need not to exceed the fraction size requirement in any normal tissue (relation (9)).

$$
\begin{gathered}
N \leq \bar{N}=\min _{i \in S_{k}, k \in V}\left\{\frac{d_{i}}{l_{k}^{\text {daily }}}, \frac{d_{\text {min }}}{l_{\text {tumor }}^{\text {daily }}}\right\} \\
N \geq \underline{N}=\max _{i \in H_{k}, k \in K}\left\{\frac{d_{i}}{u_{k}^{\text {daily }}}\right\}
\end{gathered}
$$

The number of fractions $N$ for a plan should satisfy $\underline{N} \leq N \leq \bar{N}$. If these fraction size requirements cannot be satisfied for any integral $N$, then the plan produced without consideration of the fraction size requirements is infeasible. Furthermore, a plan for which no integer number of fractions will satisfy bounds (8) and (9) on the fraction size cannot be renormalized to satisfy the fraction size requirements. Violation $\underline{N}>N$ means from (8) and (9) that

$$
\max _{i \in H_{k}, k \in K}\left\{\frac{d_{i}}{u_{k}^{\text {daily }}}\right\}>\min _{i \in S_{k}, k \in V}\left\{\frac{d_{i}}{l_{k}^{\text {daily }}}, \frac{d_{\min }}{l_{\text {tumor }}^{\text {daily }}}\right\}
$$

Thus, renormalizing the plan by scaling down the beamlet intensities, $x_{j g}$, and thus total doses $d_{i}$ by factor $\lambda>0$, has only the effect of multiplying both sides of (10) by the same $\lambda>0$. The infeasibility would remain.

\subsection{Simultaneous Method}

Our proposed Simultaneous Method, unlike the earlier approach, optimizes over both the cumulative and per fraction dose limits at the same time, where the total number of fractions are included explicitly [31]. The complete problem was specified as a linear or mixedinteger program, in which doses in each tissue must fall within an allowed range over each treatment session, and the cumulative dose distribution across each structure must meet the specified dose, dose-volume and homogeneity limits.

In order to achieve this, objective function (2), homogeneity limits (3) or (4)-(5) combined with cumulative constraints (6) and (7) are retained, and new constraints (11)-(13) are introduced as follows:

$$
\begin{gathered}
d_{i} \leq N \times u_{k}^{\text {daily }} \quad \forall i \in H_{k}, k \in K \\
d_{i} \geq N \times l_{k}^{\text {daily }} \quad \forall i \in S_{k}, k \in V \\
d_{i} \geq N \times l_{\text {tumor }}^{\text {daily }} \quad \forall i \in T
\end{gathered}
$$

Here, $N$ represents the total integer number of treatment sessions. Upper and the lower fraction doses and limits for tumor, secondary targets, and healthy tissues along with per-fraction doses are denoted by the superscript "daily" assuming one fraction is delivered per day. 
The effect of constraints (11)-(13) is to ensure that total doses $d_{i}$ accommodate $N$ times the applicable per-fraction limit on each tissue. Then a feasible fraction plan can be obtained simply by dividing all doses (equivalently intensities) by $N$. We choose to treat $N$ as an integer variable and optimize the number of fractions.

\subsection{Two-Phase Planning with Simultaneous Method}

In the two-phase planning approach, there are different plans and numbers of fractions for the two phases. We extend our Simultaneous Method by adding subscripts $e=1,2$ to distinguish doses, intensities, and the numbers of fractions for the first and second periods respectively. Table 1 details the mixed-integer program.

In Table 1, constraint (15) puts an upper bound on cumulative healthy tissue dose, (16) enforces tumor homogeneity, (17) and (18) limit the maximum healthy tissue dose per each fraction of the two phases, and (19)(20) set maximum tumor fraction size in each phase. Constraints (21) and (22) applies cumulative and per-fraction constraints on secondary targets only in phase 1 . It should be noted that the only integer variables of the above method are $N_{1}$ and $N_{2}$, which makes the mixed-integer linear programming (MILP) formulation a relatively easy one to handle. The method is tested with the same prostate case and compared to uniform fractionation scenario.

\section{Case Studies}

CPLEX with branch and bound algorithm was employed for the following test case. The points for optimization were distributed throughout the contours, determined randomly within a structure volume for computation efficiency for optimization rather than employing a uniform point set, yet highly concentrated especially within the target and the critical structures of interest. [32-36]. For each structure, we presented numbers of points used in sampling and computed the mean distances to the nearest neighbor point. Further details are provided below. The influence matrix was calculated by using the standard radiation therapy software GRATIS [37].

\subsection{Effect of Including Fraction Size Post-Optimally with Cumulative First}

The effect of Cumulative First Method, i.e. not including the fraction size constraints in optimization is examined for a case of carcinoma of prostate. The data set consisted of $23 \mathrm{CT}$ slices of which 5 are illustrated in Figure 1. The prostate case contained a primary target volume identified as the prostate gland, a secondary target volume consisting of the seminal vesicles, and four healthy tissue structures, namely the bladder, two femoral heads, rectum and all other surrounding unspecified normal tissue. Number of sample points used (the mean distance to the nearest neighbor point) is $400(0.44 \mathrm{~cm})$ for bladder, $1000(0.38 \mathrm{~cm})$ for both femoral heads together, $1000(0.28 \mathrm{~cm})$ for rectum, $465(1.4 \mathrm{~cm})$ for the remaining unspecified tissue, $786(0.29 \mathrm{~cm})$ for PTV1 (Planning Target Volume 1), and $252(0.24 \mathrm{~cm})$ for the seminal vesicles. The prescriptions for both cumulative and fraction doses are given in Table 2.

Table 1. Two-phase planning with simultaneous model.

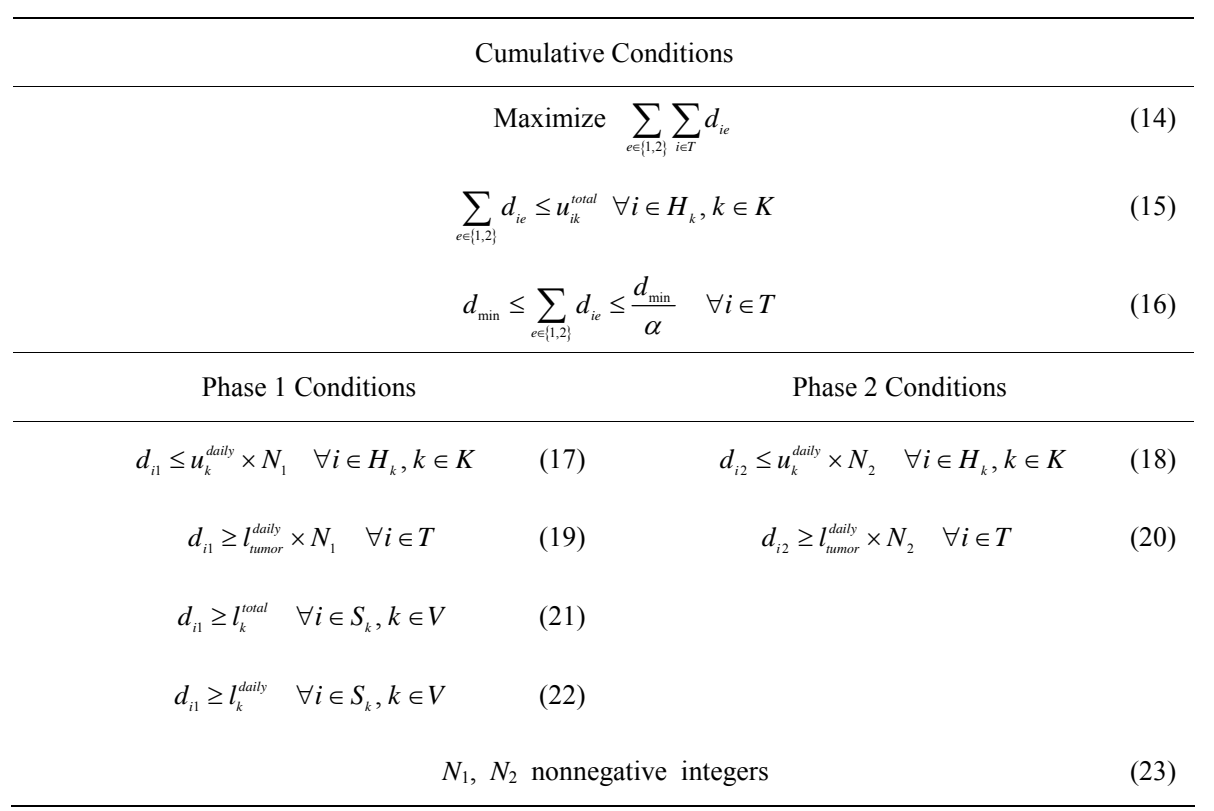


Table 2. Prostate case study prescription.

\begin{tabular}{lcccc}
\hline \multicolumn{1}{c}{ Site } & $\begin{array}{c}\text { Volume } \\
\text { Definition }\end{array}$ & $\begin{array}{c}\text { Cumulative Dose } \\
\text { Objective (Gy) }\end{array}$ & $\begin{array}{c}\text { Dose/fx } \\
\text { Objective (Gy) }\end{array}$ & $\begin{array}{c}\text { Restricted } \\
\text { Volume }\end{array}$ \\
\hline PTV1 & $\leq 84$ & $\geq 2$ & $100 \%$ \\
Seminal Vesicles & PTV2 & $\leq 84$ & $\geq 2$ & $100 \%$ \\
Bladder & & $\geq 56$ & $\leq 2$ & $100 \%$ \\
& Normal & $\leq 85$ & & $80 \%$ \\
Femoral Heads (2) & & $\leq 80$ & $\leq 2$ & $100 \%$ \\
& Normal & $\leq 72$ & & $60 \%$ \\
Rectum & & $\leq 50$ & $\leq 2$ & $100 \%$ \\
Other unspecified normal tissue & Normal & $\leq 85$ & $\leq 2$ & $100 \%$ \\
\hline
\end{tabular}

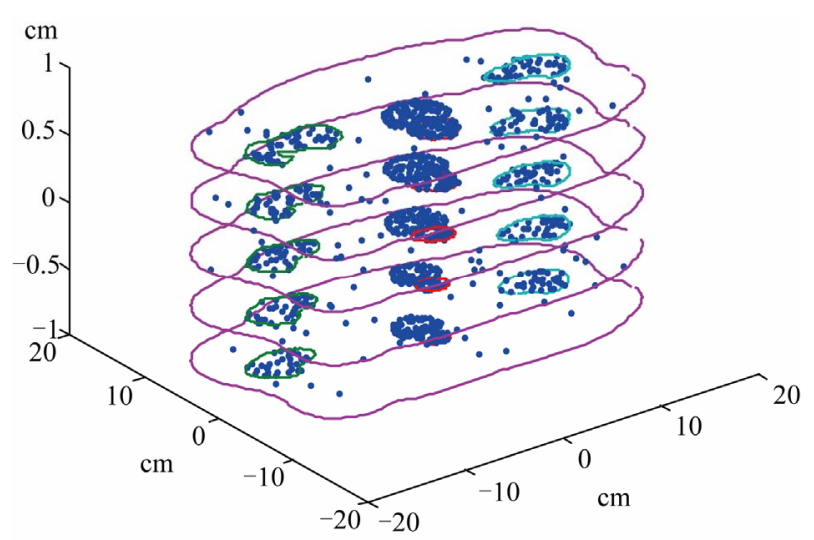

Figure 1. Outlines of 5 of the 23 transverse slices of the prostate case taken through the inferior portion of the treatment volume, with optimization sample points shown within structures of interest. The densest collection of points is taken within and on the boundary of the prostate target. Also shown are samplings from the two femurs, the rectum, and the unspecified normal tissue within the external surface.

The prescription includes a set of limits on the maximum doses that can be received in a structure as well as partial volume limits placed on the bladder, rectum and femoral heads. Those constraints are reduced to pointby-point upper limits on those tissues by protecting the points farthest from the tumor as explained in Subsection 2.1. Table 2 also includes upper or lower bounds on the doses that can be given to structures of interest each fraction. A lower bound on the dose per fraction to PTV1 and PTV2 (Planning Target Volumes $1 \&$ 2), and an upper bound on the dose per fraction to any normal tissue structure are set at 2 Gy. Tumor dose homogeneity is required as in constraints (4) and (5) by a minimum dose limit of 79 Gy and maximum dose limit of 84 Gy to PTV1. In addition to the minimum cumulative dose limit on secondary target, a maximum dose limit of $84 \mathrm{~Gy}$ is also assigned to ensure that the surrounding structures of the tumor remain below the maximum tumor dose. The number of fields (beam angels) used in the plan is 9, with the angles pre-specified.

A treatment plan was prepared first with Cumulative First Method without regarding to the fraction size limits, but only regarding to the cumulative dose limits, using the optimization model described in Subsection 2.1. The resulting dose-volume histograms (DVH) for the target, bladder and rectum are shown in Figure 2. The DVHs show the dose received by the volume percentage of the critical structures. All of the extreme dose and partial dose volume conditions expressed in Table 2 were satisfied over the points employed in the optimization routine.

Table 3 shows the values obtained for the extreme doses delivered to target or normal tissues alongside the fraction size and cumulative dose limits which apply to them. The final column shows the number of fractions (treatment sessions) that can be prescribed under each separate condition for a normal tissue or target structure, found by applying relations (8) and (9), and substituting D05 and D95 values for the maximum and minimum doses. Here, D05 and D95 denote the minimum dose delivered to the hottest $5 \%$ and $95 \%$ of the structure, respectively.

Scanning Table 3 shows that the produced plan meets the required cumulative dose conditions, but a number of fractions cannot be determined such that all the fraction 
size requirements are met. The unspecified tissue, bladder and rectum conditions require more fractions to be delivered than the maximum allowed by the condition on the secondary target (PTV2), and bladder and rectum require more fractions than allowed by the primary target. For example, the bladder requires a minimum of 42 fractions to satisfy its fraction size goals with this plan, while the PTV2 requires a maximum of 28 fractions. Renormalizing this plan to satisfy the fraction size requirements is not possible for any number of treatment sessions as explained above.

Instead of trying to fractionate the plan after the optimization, the fractionation was imposed as part of the optimization problem for the same case using the Simultaneous Method of Subsection 2.2. The results are given in the form of dose-volume histograms for target, bladder and rectum in Figure 3. The total number of fractions, $N$, is optimized and found that the plan obtained with both fraction size and cumulative dose limits can be delivered in 41 equal fractions.

Table 4 compares the relevant statistics on the extreme doses received in targets and healthy tissues under with and without fraction size limits. With either model, both the extreme dose values and the partial volume doses satisfied their required limits. As can be seen, the minimum primary target dose in the model that satisfied the fraction size limits was only $1.2 \%$ less ( 83.88 vs $82.91 \mathrm{~Gy})$ than the infeasible solution found by removing the fraction size requirement.

Comparing Figure 2 and Figure 3, there are no significant differences in the rectum and target dose distribution; however the bladder dose in the $60 \%-20 \%$ volume region increases when fraction size limits are included to the planning. Still, when the extreme doses are considered, the drop in the bladder and rectal doses are greater than the $0.26 \%$ drop in the primary target dose,

Table 3. Dose statistics of the prostate case obtained by optimizing with cumulative first method (Disregarding fraction constraints).

\begin{tabular}{|c|c|c|c|c|c|}
\hline Structure & Dose Statistics & $\begin{array}{c}\text { Required Cumulative } \\
\text { Dose (Gy) }\end{array}$ & $\begin{array}{c}\text { Achieved Cumulative } \\
\text { Dose (Gy) }\end{array}$ & $\begin{array}{l}\text { Dose per Fraction } \\
\text { Limit (Gy) }\end{array}$ & $\begin{array}{l}\text { Resulting Bound on } \\
\text { Number of Fractions }\end{array}$ \\
\hline Target & Min (D95) & $\begin{array}{l}\leq 84.0 \\
\geq 79.0\end{array}$ & 83.88 & $\geq 2.0$ & $\leq 41$ \\
\hline PTV2 & Min (D95) & $\begin{array}{l}\leq 84.0 \\
\geq 56.0\end{array}$ & 56.09 & $\geq 2.0$ & $\leq 28$ \\
\hline Bladder & $\operatorname{Max}(\mathrm{D} 05)$ & $\leq 85.0$ & 83.74 & $\leq 2.0$ & $\geq 42$ \\
\hline FemHead1 & $\operatorname{Max}(\mathrm{D} 05)$ & $\leq 72.0$ & 48.50 & $\leq 2.0$ & $\geq 25$ \\
\hline FemHead2 & $\operatorname{Max}(\mathrm{D} 05)$ & $\leq 72.0$ & 47.12 & $\leq 2.0$ & $\geq 24$ \\
\hline Rectum & $\operatorname{Max}(\mathrm{D} 05)$ & $\leq 85.0$ & 82.99 & $\leq 2.0$ & $\geq 42$ \\
\hline Otherwise unspecified tissue & $\operatorname{Max}(\mathrm{D} 05)$ & $\leq 85.0$ & 63.74 & $\leq 2.0$ & $\geq 32$ \\
\hline
\end{tabular}

Table 4. Comparison of cumulative dose statistics of the prostate case given by cumulative first and simultaneous methods.

\begin{tabular}{lccc}
\hline \multicolumn{1}{c}{ Structure } & Statistics & Cumulative First Method (Gy) & Simultaneous Method (Gy) \\
\hline \multirow{2}{*}{ Target } & Max Dose (D05) & 84.00 & 84.00 \\
& Min Dose (D95) & 83.88 & 82.91 \\
PTV2 & Mean Dose & 83.97 & 83.75 \\
Bladder & Min Dose (D95) & 56.09 & 82.00 \\
FemHead1 & Max Dose (D05) & 83.74 & 81.97 \\
FemHead2 & Max Dose (D05) & 48.50 & 48.66 \\
Rectum & Max Dose (D05) & 47.12 & 50.68 \\
Otherwise unspecified tissue & Max Dose (D05) & 82.99 & 80.74 \\
\hline
\end{tabular}




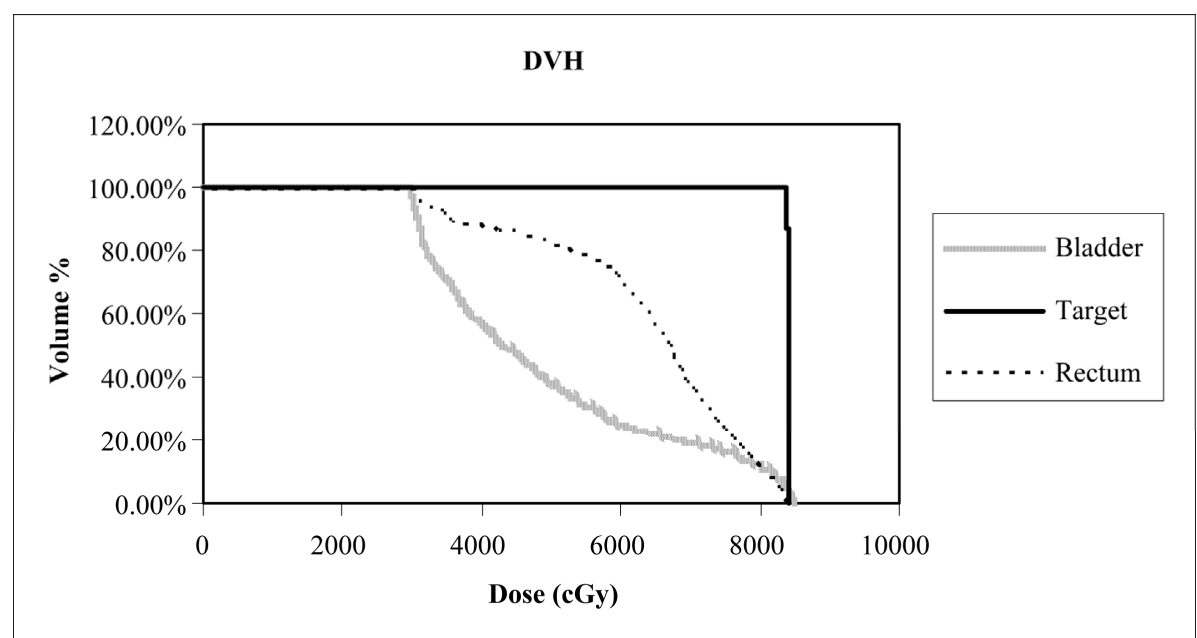

Figure 2. DVH obtained with optimization of the prostate case with cumulative first method (Disregarding fraction constraints).

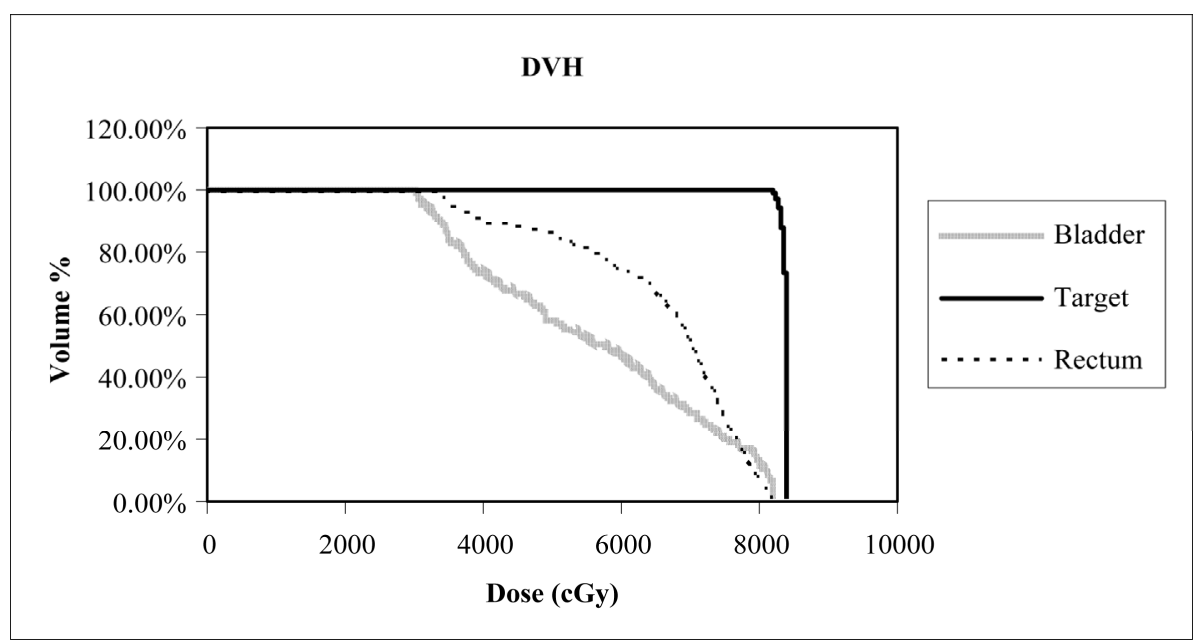

Figure 3. DVH obtained with optimization of the prostate case with fraction size and cumulative dose limits together (Simultaneous method).

drops of 1.77 Gy and 2.25 Gy respectively.

Noteworthy is the change in the dose delivered to the secondary target (PTV2). The lower dose bound for each treatment session of 2.0 Gy placed on this structure could not be satisfied simultaneously with an upper dose bound for each session of 2.0 Gy placed on normal tissues if the model that eliminated the fractionation terms were used. Once the fractionation terms were added, the minimum dose in the PTV1 fell by $0.26 \%$ but the minimum dose in the PTV2 rose from 56 Gy to $82 \mathrm{~Gy}$.

A comparison of isodose plots for the plans constructed with and without a limit on fraction sizes delivered to the critical tissues is shown in Figures 4(a) and (b). The isodose plots are very similar, showing that a deliverable plan can be obtained reaching the same cumulative doses when the fractionation considerations are included in the optimization.

\subsection{Two-Phase Planning versus Uniform Fractionation with Simultaneous Method}

The prostate case studied above with uniform fractionation with simultaneous method is further analyzed with the two-phase formulation given in Subsection 2.3. Both cumulative and fraction size limits are still enforced, however the minimum fraction size limits on PTV2 is removed after the minimum cumulative dose limit of 56 Gy is reached in each and every point of this structure as in the model in Table 1.

The results are given in Table 5. The optimized numbers of fractions for the first and second phases are 28 and 13 respectively, matching the total of 41 equal fractions with the previous plan. Keeping in mind that there is a minimum and maximum dose limit on the primary target PTV1, insignificant difference is observed on the 


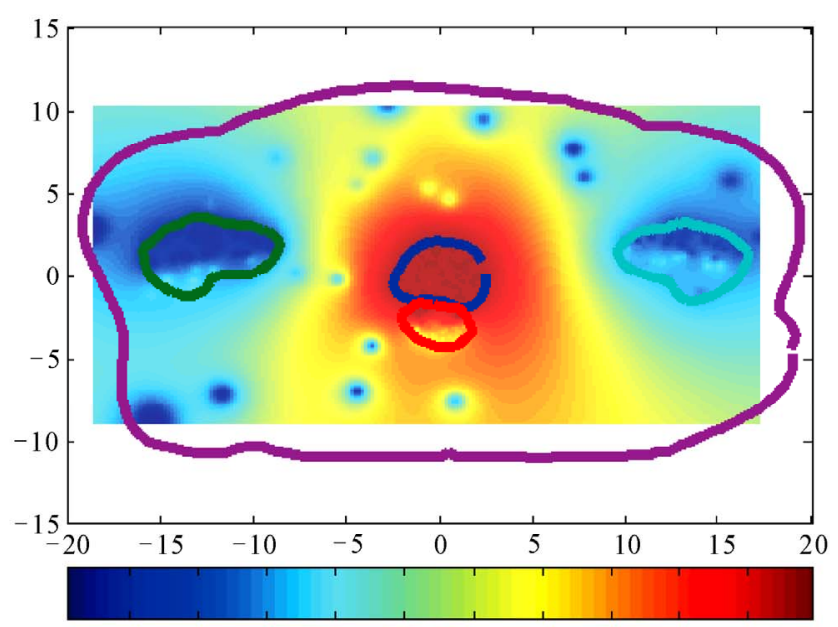

3500400045005000550060006500700075008000

(a)

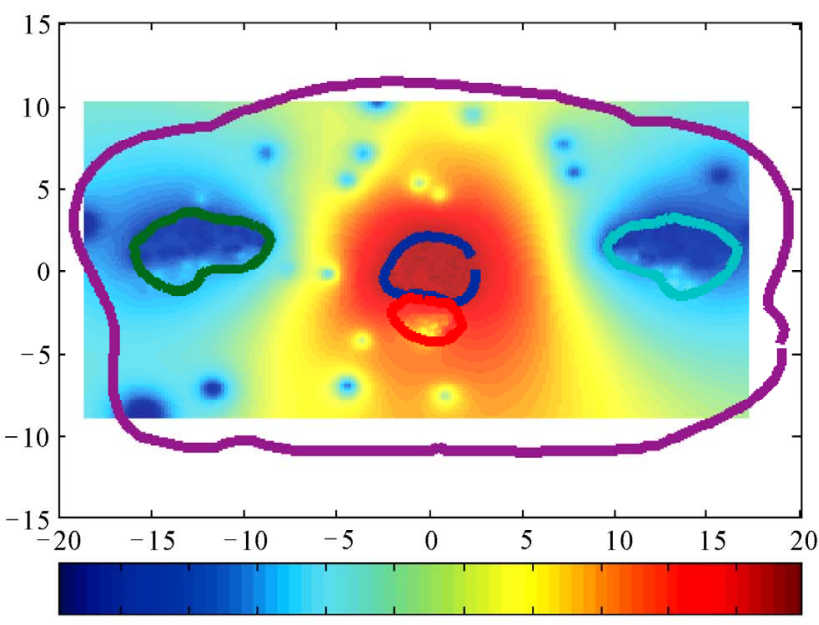

3500400045005000550060006500700075008000

(b)

Figure 4. (a) Isodose surfaces for the prostate plan obtained with cumulative first method; (b) Isodose surfaces for the prostate plan obtained with simultaneous method.

Table 5. Comparative results of the prostate case with the two-phase model.

\begin{tabular}{lccc}
\hline \multicolumn{1}{l}{ Structure } & Statistics & Uniform Fractionation with & Two-Phase Model (Gy) \\
\hline \multirow{2}{*}{ Target } & Max Dose (D05) & 84.00 & 84.00 \\
& Min Dose (D95) & 82.91 & 82.92 \\
& Mean Dose & 83.75 & 83.76 \\
PTV2 & Max Dose (D05) & 84.00 & 83.46 \\
& Min Dose (D95) & 82.00 & 64.90 \\
Bladder & Mean Dose & 82.98 & 75.70 \\
FemHead1 & Max Dose (D05) & 81.97 & 81.73 \\
FemHead2 & Max Dose (D05) & 48.66 & 47.38 \\
Rectum & Max Dose (D05) & 50.68 & 49.09 \\
Otherwise unspecified tissue & Max Dose (D05) & 80.74 & 80.37 \\
& Max Dose (D05) & 63.06 & 64.59 \\
\hline
\end{tabular}

PTV1 dose distribution between the two methods. However, the decrease in the PTV2 seminal vesicles is quite noteworthy. It can be observed further in detail in the dose-volume histograms given in Figure 5 that delivering the plan in two different phases helps to spare the vesicles from significant amount of radiation. Unnecessary exposure to radiation in those irradiated areas increases the potential of complications at the end of the treatment, although there is a minimum cumulative limit on the secondary targets; it is not the intention of the planner to radiate them above 56 Gy. Furthermore, although the extreme values do not show much difference between the two approaches, the dose volume histograms given in Figure $\mathbf{6}$ demonstrate that removing fraction size requirement from seminal vesicles during the second phase of the treatment helps to reduce the bladder dose on average significantly and rectum exposure slightly as well. Although the extreme dose limits on these two organs at risk are almost the same with the two methods, the doses at different volume percentages are quite far apart.

The isodose surfaces given in Figure 7 for the two approaches provide another tool to observe the improvement with the two-phase model. The plots on the left 


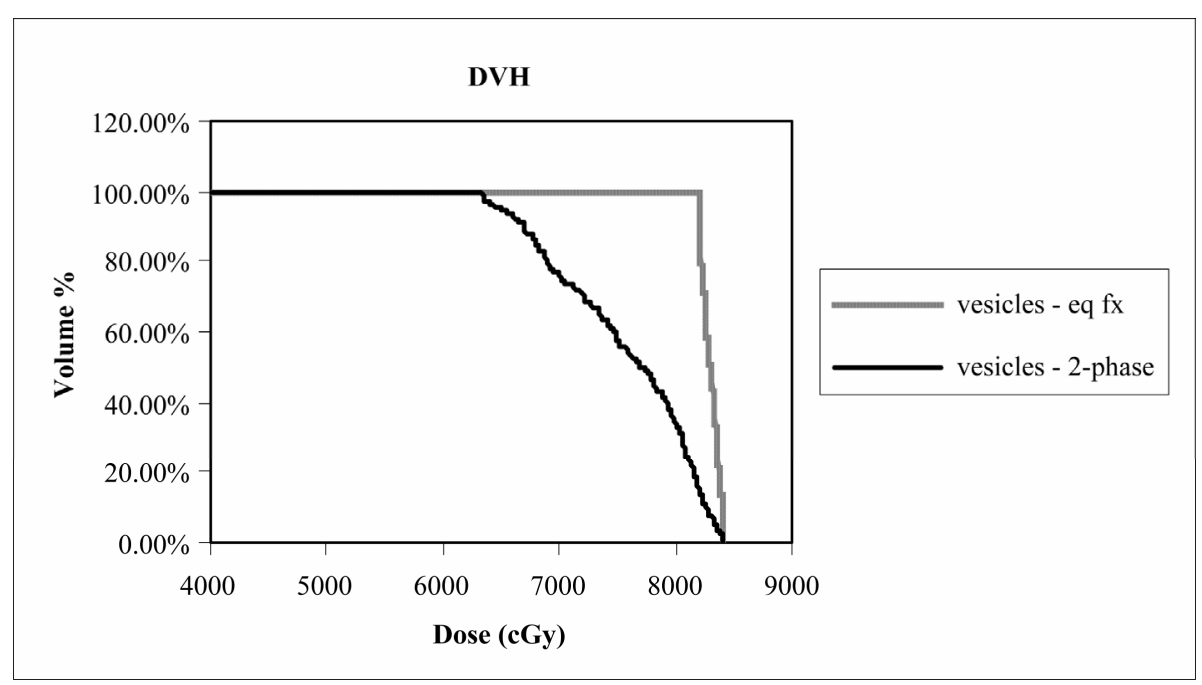

Figure 5. Comparison of DVH of vesicles for uniform fractionation and two-phase simultaneous models.

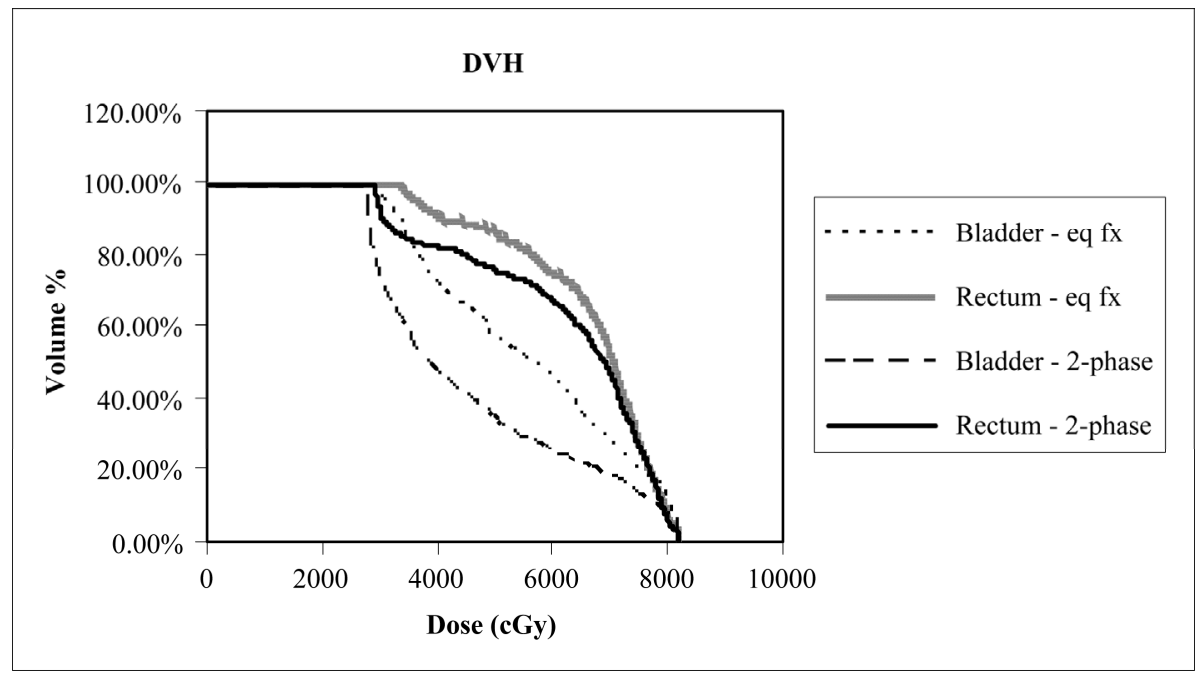

Figure 6. Comparison of DVH of bladder and rectum for uniform fractionation and two-phase simultaneous models.

correspond to the uniformly fractionated plan with the simultaneous method, while the ones on the right are obtained with two phase planning. The color scales on all four plots are kept the same for fair comparison. Although there is no apparent difference on the transverse slice at $\mathrm{z}=0$, the second slice at $\mathrm{z}=4.4$ which includes the bladder, rectum and the seminal vesicles demonstrate the decrease in radiation exposure in all parts of the irradiated volume.

\section{Discussions and Conclusions}

In this paper, the problem of producing an IMRT plan that meets fraction size and cumulative dose limits on tumor and normal tissues simultaneously is addressed. The common approach of employing the cumulative dose limits alone and considering fractionation as a post-op- timization stage, i.e. The Cumulative First approach, was demonstrated on a prostate case for which the dose distribution planned without considering fraction size requirements could not subsequently be partitioned into uniform sessions so as to satisfy the limits. It was observed that a plan that is feasible with respect to the accumulated doses may no longer remain feasible with uniform fractions once upper and lower fraction size limits are specified. The objective obtained upon imposing fraction size and cumulative limits was only $0.26 \%$ less than that which could be found even if no fraction size requirements were posed, a difference smaller than the uncertainty in the delivered dose or in the minimum dose that can be delivered in a treatment session. Hence, both cumulative and fraction size limits could be used in the planning and the fraction size requirements could be accommodated without materially losing the level of dose 


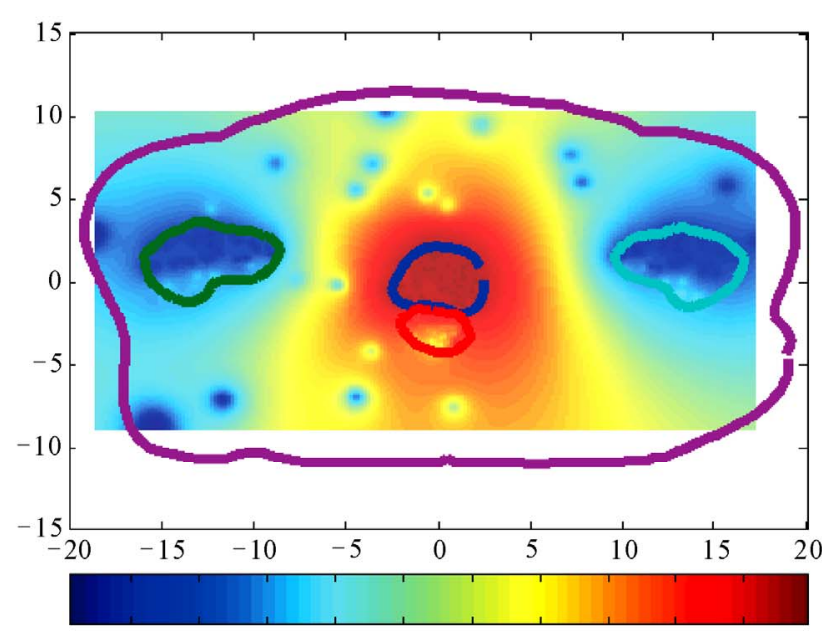

3500400045005000550060006500700075008000

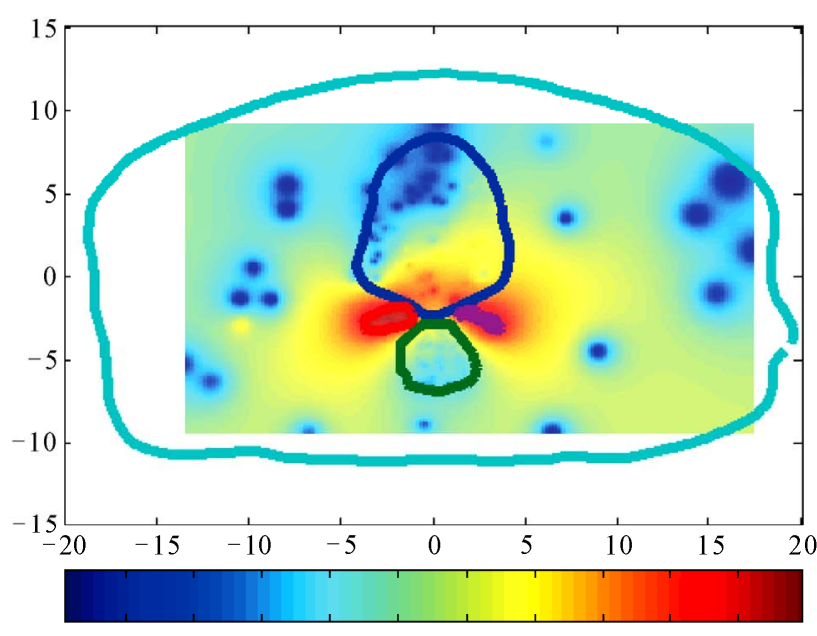

3500400045005000550060006500700075008000

(a)

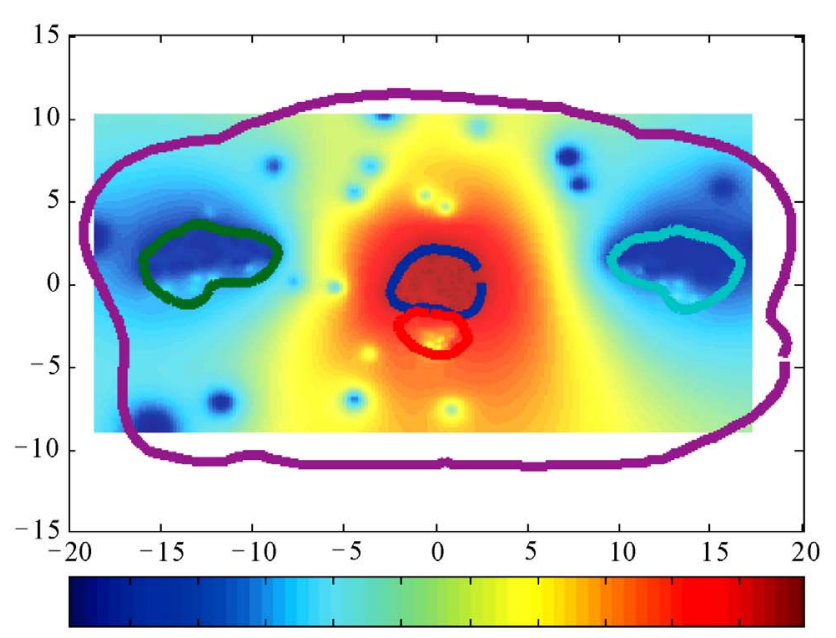

3500400045005000550060006500700075008000

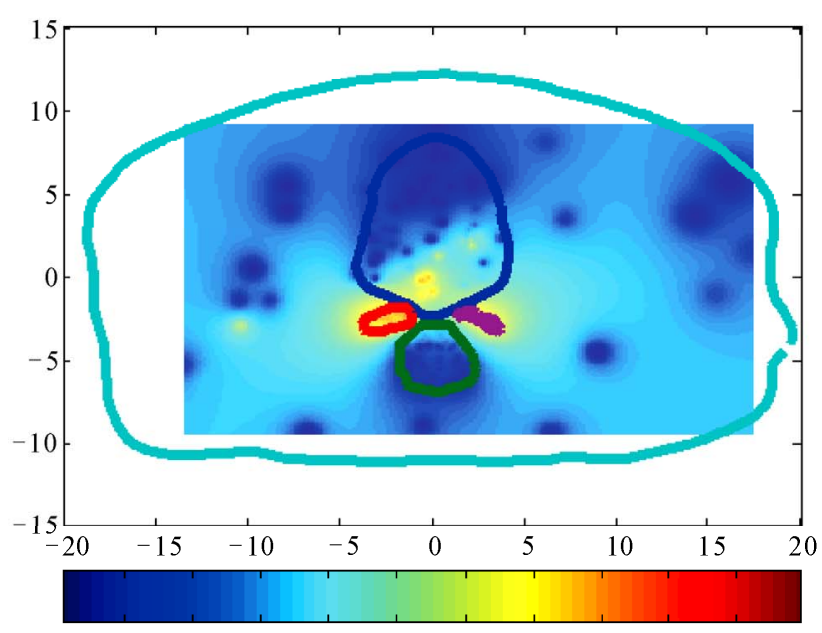

3500400045005000550060006500700075008000

(b)

Figure 7. Isodose surfaces for transverse slices of $z=0$ and $z=4.4$ prostate case with (a) Simultaneous uniform fractionation (b) Simultaneous two-phase planning.

escalation which can be achieved with IMRT.

In operations research, it is common practice to represent a problem with the least constraints possible by leaving dependent equations out to reduce solution time. However, as the experience of this research showed, the cumulative dose and fraction size limits are neither dependent nor redundant, and none of them should be eliminated from the formulation. Further, an analysis of bounds proved that no renormalization of the prescription would improve the plans developed based on the cumulative limits alone.

In the paper, it was also questioned if Simultaneous IMRT approach would benefit from combining with a conventional planning method: two-phase planning. An MILP formulation was proposed incorporating all cumulative and fraction size dose limits and optimizing the number of fractions for each phase. A two-phase solution to the prostate case without changing the irradiated margins was found in which the same tumor dose exposure was achieved while the dose to healthy structures like bladder and rectum were lessened and unwanted further exposure on the secondary target was avoided in comparison to the uniformly fractionated plan. Moreover, it has been our experience that in some cases in addition to sparing the healthy organs, the two-phase planning also helps to escalate the tumor dose.

One might assume that the detailed intensity modulation achieved with multi-leaf collimators or other modulation techniques might not require partitioning the problem into two phases in order to improve the quality of the treatment. Yet, it is demonstrated that, IMRT can benefit from the conventional tumor boosting approach. Results building on the simultaneous approach establish that releasing the fraction size minimum on the secon- 
dary target in a second phase of the therapy can favor tumor dose escalation or reduce the normal tissue exposure. Hence, the two-phase planning is still a method to explore in the era of detailed intensity maps.

The solution times are also compared for the prostate case. It increases from order of minutes to hours for the uniform fractionation versus the two-phase model. The main contributor to this change is the increase in the size of the problem, as the number of variables doubles and the number of constraints for the two epoch model increases as many as the total number of tissue points, which is close to four thousand in this specific case. It requires further research to employ efficient heuristics and approximations in order to benefit from the simultaneous and two-phase approaches in the clinics in practice. It should be noted that, although, the optimization method used here is linear programming, there is no reason not to think that the same conclusions could be reached when this analysis of simultaneous and two-phase methods was repeated with other optimization tools.

Finally, although the two-phase approach shows promise as it is, it would be of interest to the therapy planner to investigate the possibility of allowing different beam orientations during the two phases of the therapy.

\section{Acknowledgements}

We would like to thank Seda Gumrukcu for her contribution in managing submissions of this work.

\section{References}

[1] Q. Wu, M. Manning, R. Schmidt-Ulrich and R. Mohan, "The Potential for Sparing of Parotids and Escalation of Biologically Effective Dose with Intensity-Modulated Radiation Treatments of Head and Neck Cancers: A Treatment Design Study," International Journal of Radiation Oncology, Biology, Physics, Vol. 46, No. 1, 2000, pp. 195-205. doi:10.1016/S0360-3016(99)00304-1

[2] A. I. Blanco and K. S. C. Chao, "Intensity-Modulated Radiation Therapy and Protection of Normal Tissue Function in Head and Neck Cancer," In: C. A. Perez and L. W. Brady, Eds., Principles and Practice of Radiation Oncology Updates, Lippincott Williams \& Wilkins Healthcare, New York, 2002, pp. 2-12.

[3] M. Langer, R. Brown, M. Urie, J. Leong, M. Stracher and J. Shapiro, "Large Scale Optimization of Beam Weights under Dose-Volume Restrictions," International Journal of Radiation Oncology, Biology, Physics, Vol. 18, No. 4, 1990, pp. 887-893. doi:10.1016/0360-3016(90)90413-E

[4] M. Langer, P. Kijewski, R. Brown and C. Ha, "The Effect on Minimum Tumor Dose of Restricting Target-Dose Inhomogeneity in Optimized Three-Dimensional Treatment of Lung Cancer," Radiotherapy and Oncology, Vol. 21, No. 4, 1991, pp. 245-256.

\section{doi:10.1016/0167-8140(91)90049-M}

[5] M. Langer, E. K. Lee, J. O. Deasy, R. L. Rardin and J. A. Deye, "Operations Research Applied to Radiotherapy, An NCI-NSF-Sponsored Workshop," International Journal of Radiation Oncology, Biology, Physics, Vol. 57, No. 3 , 2003, pp. 762-768. doi:10.1016/S0360-3016(03)00720-X

[6] E. K. Lee, T. Fox and I. Crocker, "Integer Programming Applied to Intensity-Modulated Radiation Treatment Planning," Annals of Operations Research, Vol. 119, No. 1-4, 2003, pp. 165-181. doi:10.1023/A:1022938707934

[7] E. K. Lee, T. Fox and I. Crocker, "Simultaneous Beam Geometry and Intensity Map Optimization in Intensity-Modulated Radiation Therapy," International Journal of Radiation Oncology, Biology, Physics, Vol. 64, No. 1, 2006, pp. 301-320. doi:10.1016/j.ijrobp.2005.08.023

[8] H. E. Romeijn, R. K. Ahuja, J. F. Dempsey, A. Kumar and J. G. Li, "A Novel Linear Programming Approach to Fluence Map Optimization for Intensity Modulated Radiation Treatment Planning," Physics in Medicine and Biology, Vol. 48, No. 21, 2003, pp. 3521-3542. doi: $10.1088 / 0031-9155 / 48 / 21 / 005$

[9] H. E. Romeijn, R. K. Ahuja and J. F. Dempsey, “A New Linear Programming Approach to Radiation Therapy Treatment Planning Problems," Operations Research, Vol. 54, No. 2, 2006, pp. 201-216. doi:10.1287/opre.1050.0261

[10] F. Preciado-Walters, R. L. Rardin, M. P. Langer and V. Thai, "A Coupled Column Generation, Mixed Integer Approach to Optimal Planning of Intensity Modulated Radiation Therapy for Cancer," Mathematical Programming, Vol. 101, No. 2, 2004, pp. 319-338. doi:10.1007/s10107-004-0527-6

[11] A. Tuncel, "Methods and Algorithms for Radiation Therapy Optimization under Dose-Volume Restrictions," Ph.D. Dissertation, Purdue University, West Lafayette, 2008.

[12] F. Preciado-Walters, M. P. Langer, R. L. Rardin and V. Thai, "Column Generation for IMRT Cancer Therapy Optimization with Implementable Segments," Annals of Operations Research, Vol. 148, No. 1, 2006, pp. 65-79. doi:10.1007/s10479-006-0080-1

[13] H. E. Romeijn, R. K. Ahuja, J. F. Dempsey and A. Kumar, "A Column Generation Approach to Radiation Therapy Treatment Planning Using Aperture Modulation," SIAM Journal on Optimization, Vol. 15, No. 3, 2005, pp. 838-862. doi:10.1137/040606612

[14] S. Webb, "Optimization by Simulated Annealing of ThreeDimensional Conformal Treatment Planning for Radiation Fields Defined by a Multileaf Collimator," Physics in Medicine and Biology, Vol. 36, No. 9, 1991, pp. 12011226. doi:10.1088/0031-9155/36/9/004

[15] S. M. Morril, R. G. Lane and I. I. Rosen, "Constrained Simulated Annealing for Optimized Radiation Therapy," Computer Methods and Programs in Biomedicine, Vol. 33, No. 3, 1990, pp. 135-144. doi:10.1016/0169-2607(90)90035-8

[16] G. S. Mageras and R. Mohan, "Application of Fast Simulated Annealing to Optimization of Conformal Ra- 
diation Treatments," Medical Physics, Vol. 20, No. 3, 1993, pp. 639-647. doi:10.1118/1.597012

[17] M. Langer, S. Morrill, R. Brown, O. Lee and R. Lane, “A Comparison of Mixed-Integer Programming and Fast Simulated Annealing for Optimizing Beam Weights in Radiation Therapy," Medical Physics, Vol. 23, No. 6, 1996, pp. 957-964. doi:10.1118/1.597857

[18] M. Langer, S. Morrill, R. Brown, O. Lee and R. Lane, "A Genetic Algorithm for Generating Beam Weights," Medical Physics, Vol. 23, No. 6, 1996, pp. 965-971. doi:10.1118/1.597858

[19] G. A. Ezzel, "Genetic and Geometric Optimization of Three-Dimensional Radiation Therapy Treatment Planning," Medical Physics, Vol. 23, No. 3, 1996, pp. 293305. doi: $10.1118 / 1.597660$

[20] X. Wu, Y. Zhu, J. Dai and Z. Wang, "Selection and Determination of Beam Weights based on Genetic Algorithms for Conformal Radiotherapy Treatment Planning," Physics in Medicine and Biology, Vol. 45, No. 9, 2000, pp. 2547-2558. doi:10.1088/0031-9155/45/9/308

[21] P. S. Cho, S. Lee, R. J. Marks, S. Oh, S. G. Sutlief and M. H. Phillips, "Optimization of Intensity Modulated Beams with Volume Constraints Using Two Methods: Cost Function Minimization and Projections onto Convex Sets," Medical Physics, Vol. 25, No. 4, 1998, pp. 435443. doi:10.1118/1.598218

[22] D. H. Hristov and B. G. Fallone, "A Continuous Penalty Function Method for Inverse Treatment Planning," Medical Physics, Vol. 25, No. 2, 1998, pp. 208-223. doi:10.1118/1.598183

[23] S. V. Spirou and C. S. Chui, "A Gradient Inverse Planning Algorithm with Dose-Volume Constraints," Medical Physics, Vol. 25, No. 3, 1998, pp. 321-333. doi: $10.1118 / 1.598202$

[24] Q. Wu and R. Mohan, "Algorithms and Functionality of an Intensity Modulated Radiotherapy Optimization System," Medical Physics, Vol. 27, No. 4, 2000, pp. 701711. doi:10.1118/1.598932

[25] P. Cheung, K. Sixel, G. Morton, D. A. Loblaw, R. Tirona, G. Pang, R. Choo, E. Szumacher, G. Deboer and J. P. Pignol, "Individualized Planning Target Volumes for Intrafraction Motion during Hypofractionated IntensityModulated Radiotherapy Boost for Prostate Cancer," International Journal of Radiation Oncology, Biology, Physics, Vol. 62, No. 2, 2005, pp. 418-425.

[26] M. Guerrero, X. A. Li, L. Ma, J. Linder, C. Deyoung and B. Erickson, "Simultaneous Integrated Intensity-Modulated Radiotherapy Boost for Locally Advanced Gynecological Cancer: Radiobiological and Dosimetric Considerations," International Journal of Radiation Oncology,
Biology, Physics, Vol. 62, No. 3, 2005, pp. 933-939. doi:10.1016/j.ijrobp.2004.11.040

[27] M. L. Cavey, J. E. Bayouth, M. Colman, E. J. Endres and G. Sanguineti, "IMRT to Escalate the Dose to the Prostate While Treating the Pelvic Nodes," Strahlenther Onkol, Vol. 181, No. 7, 2005, pp. 431-441.

doi:10.1007/s00066-005-1384-9

[28] X. A. Li, J. Z. Wang, P. A. Jursinic, C. A. Lawton and D. Wang, "Dosimetric Advantages of IMRT Simultaneous Integrated Boost for High-Risk Prostate Cancer," International Journal of Radiation Oncology, Biology, Physics, Vol. 61, No. 4, 2005, pp. 1251-1257. doi:10.1016/j.ijrobp.2004.11.034

[29] R. A. Popple, P. B. Prellop, S. A. Spencer, J. F. De Los Santos, J. Duan, J. B. Fiveash and I. A. Brezovich, "Simultaneous Optimization of Sequential IMRT Plans," Medical Physics, Vol. 32, No. 11, 2005, pp. 3257-3266. doi:10.1118/1.2064849

[30] S. M. Morrill, R. G. Lane, J. A. Wong and I. I. Rosen, "Dose-Volume Considerations with Linear Programming Optimization," Medical Physics, Vol. 18, No. 6, 1991, pp. 1201-1210. doi:10.1118/1.596592

[31] D. Dink, “Approaches to 4-D Intensity Modulated Radiation Therapy Planning with Fraction Constraints," Ph.D. Dissertation, Purdue University, West Lafayette, 2005.

[32] S. M. Morrill, I. I. Rosen, R. G. Lane and J. A. Belli, "The Influence of Dose Constraint Point Placement on Optimized Radiation Therapy Treatment Planning," International Journal of Radiation Oncology, Biology, Physics, Vol. 19, No. 1, 1990, pp. 129-1241.

[33] A. Niemierko and M. Goitein, "Random Sampling for Evaluating Treatment Plans," Medical Physics, Vol. 17, No. 5, 1990, pp. 753-762. doi:10.1118/1.596473

[34] A. Niemierko and M. Goitein, "Letter to the Editor, Comments on "Sampling Techniques for the Evaluation of Treatment Plans',' Medical Physics, Vol. 20, No. 1, 1993, pp. 1377-1380. doi:10.1118/1.597103

[35] X.-Q. Lu and L. M. Chin, "Sampling Techniques for the Evaluation of Treatment Plans," Medical Physics, Vol. 20, No. 1, 1993, pp. 151-161. doi:10.1118/1.597096

[36] R. Acosta, W. Brick, A. Hanna, A. Holder, D. Lara, G. McQuilen, D. Nevin, P. Uhlig and B. Salter, "Radiotherapy Optimal Design: An Academic Radiotherapy Treatment Design System," In: J. W. Chinneck, B. Kristjansson, and M. J. Saltman, Eds., Operations Research and Cyber-Infrastructure, Springer, New York, 2009, pp. 401 425. doi:10.1007/978-0-387-88843-9 21

[37] Sherouse Systems Inc., 2011. http://www.gwsherouse.com 